\title{
Clinical and histological associations of cagA and vacA genotypes in Helicobacter pylori gastritis
}

\author{
V J Warburton, S Everett, N P Mapstone, A T R Axon, P Hawkey, M F Dixon
}

\begin{abstract}
Aims-To determine the relation among the cytotoxin associated gene $(\operatorname{cag} A)$ and vacuolating cytotoxin gene (vacA) status of Helicobacter pylori isolates, the associated clinical diseases, and the severity and pattern of chronic gastritis.

Methods-Helicobacter pylori was cultured from gastric biopsies obtained from dyspeptic patients. DNA was extracted from the isolates and the $\operatorname{cag} A$ and $\operatorname{vac} A$ status determined by the polymerase chain reaction (PCR). The prevalence of the different cag $A$ and vacA genotypes in three clinical groups, duodenal ulcer, gastric ulcer, and non-ulcer dyspepsia was compared. The histological features in sections from two antral and two corpus biopsies were graded by one blinded observer. The grades were compared with age and sex matched groups with different cagA and vacA genotypes, and with duodenal ulcers, or non-ulcer dyspepsia.
\end{abstract}

Results-Isolates from 161 patients were included. One hundred and nine $(68 \%)$ harboured a cagA+ strain and $143(89 \%)$ harboured a vacA s1 strain. The prevalence of cagA+ strains in duodenal ulcer patients (94\%) was highly significantly greater than in those with non-ulcer dyspepsia $(56 \%)$. However, of the patients infected with a cagA+ strain, almost equal numbers had non-ulcer dyspepsia or peptic ulceration. Chronic inflammation, polymorph activity, surface epithelial degeneration, atrophy, and intestinal metaplasia were all significantly more severe in the cagA+ than in the cagA- group, whereas only corpus epithelial degeneration was significantly more severe in the vacA $s 1$ group compared with the vacA s2 group. Patients infected with cagA+ strains were almost four times more likely to have antral intestinal metaplasia than cagA- patients. An antral predominant gastritis was present in duodenal ulcer patients compared with matched nonulcer dyspepsia patients, but this was not attributable to cagA or vacA status.

Conclusions-Helicobacter pylori strains showing cagA positivity and the vacA s1 genotype are associated with more severe gastritis but these virulence factors do not appear to determine the overall pattern. The pattern is closely linked to clinical disease. Therefore, it is likely that the nature of the disease complicating chronic infection is determined by host and environmental factors, while bacterial factors determine the magnitude of the risk of developing such disease. (F Clin Pathol 1998;51:55-61)

Keywords: Helicobacter pylori; cagA; vacA; chronic gastritis; peptic ulcer; intestinal metaplasia; gastric cancer

Helicobacter pylori is a major aetiological agent in a range of gastroduodenal diseases including chronic gastritis, peptic ulceration, gastric cancer, and lymphoma. Although $H$ pylori infection always results in histological gastritis, only a minority of infected subjects develop an associated clinical disease. The fact that individuals with pangastritis and multifocal atrophy are more likely to develop gastric ulcer disease and cancer, and those with antral predominant gastritis have a propensity to develop duodenal ulcers indicates an association between the pattern of $H$ pylori induced inflammation and disease outcome. This pattern could be determined by the virulence of the infecting strain or host and environmental factors.

An important virulence determinant of $H$ pylori is the vacuolating cytotoxin (VacA). This unique type of extracellular toxin is found more often in strains from patients with duodenal ulcer disease. ${ }^{1}$ Anti-VacA antibodies from such patients are found to neutralise the toxin itself, which suggests that it has an important role in vivo. ${ }^{2}$ Carriage of VacA+ strains has also been shown to increase polymorph infiltration in the antrum. ${ }^{23}$ All strains carry a single copy of the vacA gene, but a proportion do not produce the cytotoxin. ${ }^{3}$ The vacA gene is known to differ between cytotoxin producing and noncytotoxic strains, ${ }^{4}$ with three different signal sequences having been identified: $\mathrm{s} 1 \mathrm{a}, \mathrm{s} 1 \mathrm{~b}$, and s2. Strains with the s1 genotype (both $a$ and $b$ ) are able to produce cytotoxin, whereas those with the s2 genotype are not. ${ }^{5}$

A second gene that was thought initially to be implicated in cytotoxin production is the cytotoxin associated gene (cagA). ${ }^{6}$ Previous work indicates that about $60 \%$ of strains possess the cagA gene, of which nearly all express the immunodominant gene product, the CagA protein. ${ }^{7}$ It is now appreciated that the cagA gene product is not itself a virulence factor, but that the gene is a member of a $40 \mathrm{~kb}$ cluster of genes (a "pathogenicity island"), some of which do contribute to bacterial pathogenicity. ${ }^{8}$ Thus, cagA + strains induce inflammatory cytokines (especially interleukin 8 (IL-8)) in vivo more readily than do cagAstrains. ${ }^{9}$ Interestingly, anti-CagA antibodies are significantly more prevalent in patients with duodenal ulcers ${ }^{10}{ }^{11}$ and gastric cancer ${ }^{12}{ }^{13}$ than in patients with non-ulcer dyspepsia. Given 
that these two diseases are associated with different patterns of gastritis, it is likely that cagA is not a determinant of the pattern itself, but this has not been investigated. To examine the relation between putative virulence factors and the severity and distribution of gastritis, we endeavoured to type $H$ pylori strains with respect to their cagA and vacA gene status, and to correlate these findings with the pattern of gastritis and the disease associations present in the patient.

\section{Methods}

SOURCE AND ISOLATION OF H PYLORI

Patients attending an open access endoscopy service for the investigation of dyspepsia had mucosal biopsies taken from the antrum (four) and corpus (three). One of the antral biopsies was used for an immediate rapid urease test and, if positive, initially one antral, and later one antral and one corpus biopsy specimen were sent in transport medium for culture. The other biopsy specimens were immersed in $10 \%$ formalin and submitted for histology. The patients were asked specifically about recent use of non-steroidal anti-inflammatory drugs (NSAIDs) and antisecretory drugs $\left(\mathrm{H}_{2}\right.$ blockers and proton pump inhibitors). Patients were not included if they had been on such treatment in the preceding four weeks.

ISOLATES

Stored isolates of $\mathrm{H}$ pylori (kept at $-70^{\circ} \mathrm{C}$ ) from antral biopsies alone were available from 39 patients while fresh isolates from both antrum and body biopsies were obtained from 122 patients. For all experiments, the $H$ pylori type strain NCTC 11637 was used as a control, to compare with other published studies. Isolates were grown up on selective media (GC agar base; Oxoid, Basingstoke, UK), supplemented with lysed blood, L-glutamine, cocarboxylase, ferric nitrate, L-cystine, and dextrose (GC Supplement; Oxoid). The medium also contained the following antibiotics: vancomycin $(3 \mu \mathrm{g} / \mathrm{ml})$, polymyxin B $(6 \mu \mathrm{g} / \mathrm{ml})$, amphotericin $(1 \mathrm{mg} / \mathrm{ml})$, and trimethoprim $(6.5 \mu \mathrm{g} / \mathrm{ml})$. Plates were incubated at $37^{\circ} \mathrm{C}$ in a gas mixture of $10 \% \mathrm{CO}_{2}, 5 \%$ oxygen balanced with nitrogen for 3-7 days. Isolates were identified as $H$ pylori if they were catalase, oxidase, and urease positive, with the appearance of Gram negative curved bacilli. Isolates were harvested for storage in Brain Heart Infusion (Oxoid) containing $20 \%$ glycerol and stored at $-70^{\circ} \mathrm{C}$.

\section{HISTOLOGY}

Sections were stained by haematoxylin and eosin, alcian blue-periodic acid Schiff, and modified Giemsa. Only cases with two adequately sized biopsies of both the antral and oxyntic (corpus) mucosa were accepted for histological assessment. Cases were assessed by a single blinded observer, scoring the following features from 0-3 according to the updated Sydney System ${ }^{14}$ for both antral and corpus biopsies: chronic inflammation, polymorphonuclear activity (neutrophil infiltration), surface epithelial degeneration, atrophy, intestinal metaplasia, and $H$ pylori colonisation density.
The degree of antral predominance for these features was assessed by subtracting corpus scores from antral scores for each patient.

\section{DNA EXTRACTION}

DNA was extracted from the fresh isolates before storage at $-70^{\circ} \mathrm{C}$ and after two passages for stored isolates. Three colonies were picked from a plate of $H$ pylor $i$ with a sterile toothpick and suspended in $50 \mu \mathrm{l}$ of sterile, double distilled water. Tubes were then boiled at $95^{\circ} \mathrm{C}$ for five minutes and $2 \mu \mathrm{l}$ of a $1 / 5$ dilution of this extract (containing $\sim 100 \mathrm{ng}$ of DNA) was used as template to determine cagA prevalence and the signal sequence of the vacA gene by the polymerase chain reaction (PCR).

POLYMERASE CHAIN REACTION

Detection of $\operatorname{cag} A$

A cagA fragment of 349 base pairs was amplified from a $50 \mu \mathrm{l}$ reaction mixture containing the following: $3 \mathrm{mM} \mathrm{MgCl}_{2}, 1 \times$ reaction buffer (Advanced-Biotechnologies, Surrey, UK), $200 \mu \mathrm{M}$ of each dNTP (Boehringer Mannheim, Mannheim, Germany), and 0.5 U of Thermoprime (Advanced Biotechnologies). Fifty picomoles of primers $\mathrm{F} 1$ and $\mathrm{B} 1^{7}$ were used and the whole mixture was overlaid with $\sim 30 \mu$ of sterile mineral oil.

Determination of vacA signal sequence

The reaction mixture contained the following: $2 \mathrm{mM} \mathrm{MgCl}{ }_{2}, 1 \times$ reaction buffer (Advanced Biotechnologies), $150 \mu \mathrm{M}$ of each dNTP (Boehringer Mannheim), $0.5 \mathrm{U}$ of Thermoprime (Advanced Biotechnologies), 50 pmols of primers VAR-1 and VAR- $2,{ }^{5}$ made up to $50 \mu \mathrm{l}$ with double distilled water, and overlaid with $\sim 30 \mu \mathrm{l}$ of sterile mineral oil. Standard specimen negative controls (water) were included.

\section{Reaction conditions}

For both PCRs: $94^{\circ} \mathrm{C}$ for five minutes, $60^{\circ} \mathrm{C}$ for one minute, then 35 cycles of $94^{\circ} \mathrm{C}$ for one minute and $60^{\circ} \mathrm{C}$ for 30 seconds, followed by a final extension step of $72^{\circ} \mathrm{C}$ for five minutes.

\section{Viewing}

A $12 \mu \mathrm{l}$ aliquot of the PCR product was combined with $3 \mu \mathrm{l}$ of loading dye and run on a $2 \%$ agarose gel, stained with $10 \mathrm{mg}$ ethidium bromide, at $120-150$ volts for $25-45$ minutes (depending on the volume of the gel) in $1 \times$ Tris acetate EDTA buffer. Bands were viewed under ultraviolet excitation. For the vacA signal sequence PCR, a band 259 base pairs in size, corresponded to the $\mathrm{s} 1$ phenotype and a band size of 286 base pairs, indicated an s2 phenotype. $^{5}$

The presence of $H$ pylori DNA in cagAstrains was confirmed using a 16S rRNA PCR technique validated previously in this department ${ }^{15}$ (fig 1).

\section{STATISTICS}

The significance of differences in histological features between the cagA and vacA groups and the duodenal ulcer and non-ulcer dyspepsia groups was determined by comparing 


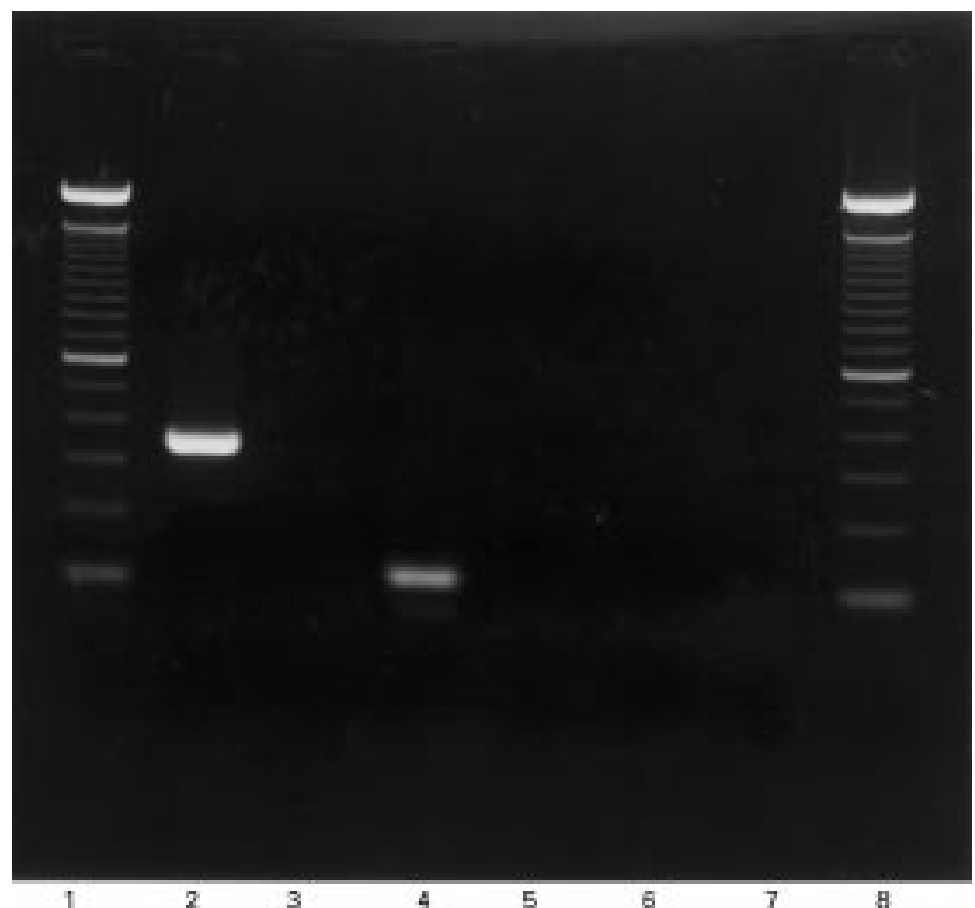

Figure 1 16S rRNA PCR technique. Lane 1, 100 bp DNA ladder; lane 2, 349 base pair cag $A$ product from cag $A+$ Helicobacter pylori; lane 3, cag $A-H$ pylori (no product); lane 4,109 base pair $16 S$ rRNA $H$ pylori product from cagA-H pylori sample; lane 5 , cag $A$ PCR negative control; lane 6, $16 S$ rRNA PCR negative control; lane 7, DNA extraction negative control; lane 8, 100 base pair DNA ladder.

individual grades using the Mann-Whitney $\mathrm{U}$ test. The $\chi^{2}$ test was used to compare the prevalences of clinical diseases between groups.

\section{Results}

A total of 161 (74 female) $H$ pylori infected patients were entered into the study. The distribution of clinical disease was as follows: 50 patients (age range, 17-77 years; median, 52 years) had current or past duodenal ulcer disease; 12 patients (age range, 33-77 years; median, 56 years) had gastric ulcer disease; and 99 patients (age range, 17-88 years; median, 54 years) had non-ulcer dyspepsia; in the latter group eight had oesophagitis, eight had duodenitis, and four had gastric erosions. The remainder had no endoscopic lesions.

The cagA and vacA status of isolates was determined on cultures from antral biopsies alone in 39 patients and from antral and corpus biopsies in 122 patients. Four of this latter group had cagA $+H$ pylori from one biopsy and cagA- from the other.

One hundred and nine patients $(68 \%)$ were infected with cagA+ strains (including four patients with both cagA + and cagA- strains),

Table 1 Prevalence of cagA and vacA s1 or s2 Helicobacter pylori

\begin{tabular}{|c|c|c|c|c|c|}
\hline & \multirow[b]{2}{*}{ Number } & \multicolumn{2}{|l|}{ cagA status } & \multicolumn{2}{|l|}{ vacA status } \\
\hline & & Positive (\%) & Negative (\%) & $\operatorname{vac} A s 1(\%)$ & vacA s2 (\%) \\
\hline Duodenal ulcer & 50 & $47(94)$ & $3(6)$ & $49(98)$ & $1(2)$ \\
\hline Gastric ulcer & 12 & $7(58)$ & $5(42)$ & $10(83)$ & $2(17)$ \\
\hline Non-ulcer dyspepsia & 99 & $55(56)$ & $44(44)$ & $84(85)$ & $15(15)$ \\
\hline Total & 161 & $109(68)$ & $52(32)$ & $143(89)$ & $18(11)$ \\
\hline
\end{tabular}

Both cagA+ and vacA s1 have a significantly greater association with duodenal ulcer than non-ulcer dyspepsia. and in 143 patients (89\%) the infecting strain was of the vacA s1 type (table 1). One hundred and four $(73 \%)$ vacA s1 types were also cagA+ compared with 36 that were cagA-. Likewise, only one vacA s2 type was cagA+ compared with 17 that were cagA-. Thus, cagA was highly significantly associated with vacA s1 $(p<0.0001)$. Of the four patients with both positive and negative cagA strains, three were of the vacA s1 type and one was of the s2 type.

cagA, vacA, AND CLINICAL DISEASE

The prevalence of cagA + strains in duodenal ulcer patients (94\%) was highly significantly greater than in those with non-ulcer dyspepsia $(56 \% ; \mathrm{p}<0.0001)$. Likewise, the distribution of vacA s1 was also significantly different in patients with duodenal ulcer disease compared with non-ulcer dyspepsia $(\mathrm{p}=0.049)$. However, of the 109 patients infected with cagA+ strains, an almost equal number have nonulcer dyspepsia as have peptic ulcer disease (55 versus 54). In contrast, only eight of 52 patients (15\%) with cagA- strains have peptic ulceration. Similarly, 59 of $143(41 \%)$ of vacA s1 strains were associated with peptic ulceration, while only three of $18(17 \%)$ patients with s2 strains had ulcers.

\section{CagA MATCHED SUBGROUP}

Fifty two patients were infected with cagAstrains and, of these, 41 patients had biopsies suitable for assessment. These patients (age range, 26-76 years; median, 54 years) were age ( \pm 5 years) and sex matched to 41 patients with cagA $+H$ pylori without regard to their clinical group (age range, 25-77 years; median, 55 years; $p=0.99$ ). The following histological features of antral mucosa were significantly $(p<0.05)$ more severe in the cagA+ group compared with the cagA- group: chronic inflammation $(\mathrm{p}=0.039)$, polymorph activity $(p=0.011)$, surface epithelial degeneration $(\mathrm{p}=0.006)$, atrophy $(\mathrm{p}=0.012)$, and intestinal metaplasia $(p=0.005)$ (table 2$)$. Corpus features of chronic inflammation $(p=0.003)$, polymorph activity $(\mathrm{p}=0.007)$, and surface epithelial degeneration $(p=0.004)$ were also significantly more severe in the cagA+ group. Of particular interest, 15 patients in the cagA+ subgroup had antral intestinal metaplasia compared with four in the cagA- group (odds ratio $=3.75 ; 95 \%$ confidence limits $=2.22-$ $6.34)$.

The prevalence of duodenal ulcers was significantly greater in the cagA+ $(34 \%)$ compared with the cagA- subgroup $(7 \%$; $\mathrm{p}=0.032$ ) but the prevalence of gastric ulcers was not.

\section{VACA MATCHED SUBGROUP}

Of 18 patients with vacA s $2 H$ pylori, 16 were suitable for histological assessment. These patients (age range, 26-76 years; median, 56 years) were age and sex matched to 16 patients with vacA s1 $H$ pylori (age range, 25-77 years; median, 55.5 years; $\mathrm{p}=0.98$ ). Corpus surface epithelial degeneration was found to be significantly $(p=0.004)$ more severe in the $\mathrm{s} 1$ group compared with the s2 group. Other features 
Table 2 Histological scores for the cagA matched groups ( $n=41$ in each group)

\begin{tabular}{|c|c|c|c|c|c|c|c|c|c|c|c|c|}
\hline & \multicolumn{6}{|c|}{ Antrum } & \multicolumn{6}{|c|}{ Corpus } \\
\hline & $C i$ & $A c$ & $S E D$ & $A t$ & $I M$ & $H p$ & $\mathrm{Ci}$ & $A c$ & $S E D$ & $A t$ & $I M$ & $H p$ \\
\hline \multicolumn{13}{|l|}{ cagAt+ } \\
\hline Mean & 1.951 & 1.366 & 1.268 & 0.780 & 0.537 & 1.610 & 1.561 & 0.878 & 0.732 & 0.537 & 0.146 & 1.244 \\
\hline Range & $1-3$ & $0-3$ & $0-3$ & $0-1$ & $0-3$ & $0-3$ & $1-3$ & $0-2$ & $0-2$ & $0-2$ & $0-3$ & $0-3$ \\
\hline $\begin{array}{l}\text { Median } \\
\text { cagA- }\end{array}$ & 2 & 1 & 1 & 1 & 0 & 2 & 1 & 1 & 1 & 0 & 0 & 1 \\
\hline Mean & 1.659 & 0.951 & 0.805 & 0.415 & 0.146 & 1.488 & 1.195 & 0.463 & 0.341 & 0.293 & 0.049 & 1.146 \\
\hline Range & $1-3$ & $0-2$ & $0-2$ & $0-2$ & $0-2$ & $0-3$ & $1-3$ & $0-2$ & $0-3$ & $0-3$ & $0-1$ & $0-3$ \\
\hline Median & 2 & 1 & 1 & 0 & 0 & 1 & 1 & 0 & 0 & 0 & 0 & 1 \\
\hline $\mathrm{z}$ & 2.065 & 2.557 & 2.754 & 2.521 & 2.811 & 0.741 & 3.011 & 2.723 & 2.870 & 1.736 & 0.863 & 1.177 \\
\hline $\mathrm{p}$ & 0.039 & 0.011 & 0.006 & 0.012 & 0.005 & 0.459 & 0.003 & 0.007 & 0.004 & 0.083 & 0.388 & 0.239 \\
\hline
\end{tabular}

Mean value is given for illustration. The individual grades for each histological feature were used in a Mann-Whitney test to explore differences.

$\mathrm{Ci}, \mathrm{Ac}$, and SED in both antrum and corpus as well as At and IM in the antrum are all significantly more severe in the cagA+ group. $\mathrm{Ci}$, chronic inflammation; Ac, polymorph activity, SED surface epithelial degeneration; At, atrophy; IM, intestinal metaplasia; Hp, Helicobacter pylori; $\mathrm{z}$, standard normal deviate; $\mathrm{p}$, significance.

Table 3 Histological scores for the vacA $s 1$ and 22 matched groups ( $n=16$ in each group)

\begin{tabular}{|c|c|c|c|c|c|c|c|c|c|c|c|c|}
\hline & \multicolumn{6}{|c|}{ Antrum } & \multicolumn{6}{|l|}{ Corpus } \\
\hline & $\mathrm{Ci}$ & $A c$ & $S E D$ & $A t$ & $I M$ & $H p$ & $C i$ & $A c$ & $S E D$ & $A t$ & $I M$ & $H p$ \\
\hline \multicolumn{13}{|l|}{ vacA s1 } \\
\hline Mean & 1.875 & 0.875 & 1.125 & 0.375 & 0.375 & 1.500 & 1.375 & 0.750 & 0.688 & 0.313 & 0.125 & 1.313 \\
\hline Range & $1-3$ & $0-2$ & $0-2$ & $0-2$ & $0-2$ & $0-3$ & $1-2$ & $0-2$ & $0-2$ & $0-1$ & $0-1$ & $1-3$ \\
\hline $\begin{array}{l}\text { Median } \\
\text { vacA s2 }\end{array}$ & 2 & 1 & 1 & 0 & 0 & 1 & 1 & 1 & 1 & 0 & 0 & 1 \\
\hline Mean & 1.438 & 0.750 & 0.625 & 0.375 & 0.063 & 11.563 & 1.250 & 0.313 & 0.125 & 0.375 & 0.063 & 1.063 \\
\hline Range & $1-3$ & $0-2$ & $0-1$ & $0-1$ & $0-1$ & $1-3$ & $1-3$ & $0-1$ & $0-1$ & $0-2$ & $0-1$ & $0-2$ \\
\hline Median & 1 & 1 & 0 & 0 & 0 & 1 & 1 & 0 & 0 & 0 & 0 & 1 \\
\hline $\mathrm{z}$ & 1.663 & 0.285 & 1.893 & -0.227 & 1.492 & -0.225 & 1.010 & 1.916 & 2.895 & -0.117 & 0.597 & 1.202 \\
\hline $\mathrm{p}$ & 0.096 & 0.776 & 0.058 & 0.820 & 0.136 & 0.822 & 0.312 & 0.055 & 0.004 & 0.907 & 0.551 & 0.229 \\
\hline
\end{tabular}

Mean value is given for illustration. The individual grades for each histological feature were used in a Mann-Whitney test to explore differences.

Corpus SED was significantly more severe in s1 compared with s2 group.

$\mathrm{Ci}$, chronic inflammation; Ac, polymorph activity, SED surface epithelial degeneration; At, atrophy; IM, intestinal metaplasia; Hp, Helicobacter pylori; $\mathrm{z}$, standard normal deviate; $\mathrm{p}$, significance.

that showed greater (but not statistically significant) severity in the $\mathbf{1}$ group were antral surface epithelial degeneration and corpus polymorph activity (table 3 ).

Duodenal ulcer, gastric ulcer, and non-ulcer dyspepsia prevalence did not differ significantly between the vacA s1 and s2 subgroups.

HISTOLOGICAL FEATURES AND CLINICAL DISEASE Twenty five patients with duodenal ulcers (age range, 26-72 years; median, 54 years) were selected randomly from the overall series and matched to 25 patients with non-ulcer dyspepsia, not including those patients with identifiable endoscopic disease such as duodenitis, gastric erosions, and oesophagitis (age range, 25-77 years; median, 56 years; $\mathrm{p}=1.0$ ), regardless of strain genotype. The prevalence of cagA or vacA type did not differ significantly between the duodenal ulcer and the non-ulcer dyspepsia subgroups. The features of corpus polymorph activity $(p=0.002)$ and surface epithelial degeneration $(p=0.015)$ were significantly more severe in patients with nonulcer dyspepsia than in those with duodenal ulceration. However, patients with duodenal ulceration demonstrated a significantly higher grade of antral chronic inflammation $(\mathrm{p}=0.008)$ and $H$ pylori density $(\mathrm{p}=0.005)$ (table 4 ). When the 12 patients with gastric ulceration were age and sex matched with nonulcer dyspepsia patients (regardless of strain type), the histological scores showed no significant differences (data not shown).

Table 4 Comparison of histological scores for age matched patients with duodenal ulcer or non-ulcer dyspepsia irrespective of cagA or vacA status ( $n=25$ in each group)

\begin{tabular}{|c|c|c|c|c|c|c|c|c|c|c|c|c|}
\hline & \multicolumn{6}{|l|}{ Antrum } & \multicolumn{6}{|l|}{ Corpus } \\
\hline & $C i$ & $A c$ & $S E D$ & $A t$ & $I M$ & $H p$ & $C i$ & $A c$ & $S E D$ & $A t$ & $I M$ & $H p$ \\
\hline \multicolumn{13}{|c|}{ Non-ulcer dyspepsia } \\
\hline Mean & 1.640 & 1.240 & 0.960 & 0.480 & 0.360 & 1.320 & 1.480 & 0.960 & 0.720 & 0.520 & 0.160 & 1.120 \\
\hline Range & $1-3$ & $0-3$ & $0-2$ & $0-2$ & $0-2$ & $0-3$ & $1-3$ & $0-2$ & $0-2$ & $0-2$ & $0-1$ & $0-3$ \\
\hline Median & 2 & 1 & 1 & 1 & 0 & 1 & 1 & 1 & 1 & 0 & 0 & 1 \\
\hline \multicolumn{13}{|c|}{ Duodenal ulcer } \\
\hline Mean & 2.120 & 1.400 & 1.080 & 0.840 & 0.320 & 1.920 & 1.240 & 0.360 & 0.280 & 0.280 & 0.120 & 1.040 \\
\hline Range & $1-3$ & $0-3$ & $0-3$ & $0-2$ & $0-3$ & $1-3$ & $1-2$ & $0-2$ & $0-2$ & $0-2$ & $0-3$ & $0-2$ \\
\hline Median & 2 & 1 & 1 & 1 & 0 & 2 & 1 & 0 & 0 & 0 & 0 & 1 \\
\hline $\mathrm{z}$ & -2.670 & -0.729 & -0.478 & -1.931 & -0.134 & -2.785 & 1.334 & 3.159 & 2.443 & 1.634 & 1.324 & 0.349 \\
\hline $\mathrm{p}$ & 0.008 & 0.466 & 0.633 & 0.054 & 0.893 & 0.005 & 0.182 & 0.002 & 0.015 & 0.102 & 0.186 & 0.727 \\
\hline
\end{tabular}

Mean value is given for illustration. The individual grades for each histological feature were used in a Mann-Whitney test to explore differences.

Antral $\mathrm{H}$ pylori density and $\mathrm{Ci}$ are significantly more severe in duodenal ulcer patients while corpus Ac and SED are more severe in non-ulcer dyspepsia patients. $\mathrm{Ci}$, chronic inflammation; Ac, polymorph activity, SED surface epithelial degeneration; At, atrophy; IM, intestinal metaplasia; Hp, Helicobacter pylori; z, standard normal deviate; $p$, significance. 
Table 5 Antral predominance (antral minus corpus grades) in patients with duodenal ulcer compared to matched non-ulcer dyspepsia patients $(n=25$ in each group)

\begin{tabular}{llllrll}
\hline & $C i$ & Ac & SED & \multicolumn{1}{c}{ At } & \multicolumn{1}{l}{ IM } & \multicolumn{1}{l}{ Hp } \\
\hline Duodenal ulcer & 0.880 & 1.040 & 0.800 & 0.560 & 0.200 & 0.880 \\
Non-ulcer dyspepsia & 0.160 & 0.280 & 0.240 & -0.040 & 0.200 & 0.200 \\
z & 3.204 & 2.888 & 2.844 & 2.387 & 0.670 & 2.725 \\
$\mathrm{p}$ & 0.001 & 0.004 & 0.005 & 0.017 & 0.503 & 0.006 \\
\hline
\end{tabular}

The mean net value is given for illustration. The individual net grades (antrum minus corpus) were used in a Mann-Whitney test to explore differences.

The net grades for all features (except IM) are significantly greater in patients with duodenal ulcer than in non-ulcer dyspepsia patients.

$\mathrm{Ci}$, chronic inflammation; Ac, polymorph activity, SED surface epithelial degeneration; At, atrophy; IM, intestinal metaplasia; Hp, Helicobacter pylori; $\mathrm{z}$, standard normal deviate; $\mathrm{p}$, significance.

Table 6 Antral predominance (antral minus corpus scores) and cagA status ( $n=41$ in each group)

\begin{tabular}{lrlllll}
\hline & \multicolumn{1}{l}{$C i$} & $A c$ & SED & At & IM & Hp \\
\hline cagA+ & 0.390 & 0.488 & 0.537 & 0.244 & 0.390 & 0.366 \\
cagA- & 0.463 & 0.488 & 0.463 & 0.122 & 0.098 & 0.341 \\
z & -0.404 & 0.140 & 0.261 & 0.669 & 2.592 & 0.524 \\
p & 0.686 & 0.889 & 0.794 & 0.504 & 0.010 & 0.600 \\
\hline
\end{tabular}

The mean net value is given for illustration. The individual net grades (antrum minus corpus) were used in a Mann-Whitney test to explore differences.

No antral predominance demonstrated apart from IM.

$\mathrm{Ci}$, chronic inflammation; Ac, polymorph activity, SED surface epithelial degeneration; At, atrophy; IM, intestinal metaplasia; Hp, Helicobacter pylori; $\mathrm{z}$, standard normal deviate; $\mathrm{p}$, significance.

INVESTIGATION OF ANTRAL PREDOMINANT

GASTRITIS

Clinical disease

When corpus scores were subtracted from antral scores to determine antral predominance in the matched duodenal ulcer and nonulcer dyspepsia patients, it was found that duodenal ulcer patients had a significant increase in net grades for all features of antral gastritis except intestinal metaplasia when compared with the net grades in non-ulcer dyspepsia patients (table 5). Of interest, non-ulcer dyspepsia patients exhibited a greater degree of atrophy in the corpus than in the antrum (mean net grade $=-0.04$ ), although this did not achieve statistical significance.

$\operatorname{cag} A$

When antral predominance was sought in relation to cagA status (for the 82 matched subjects), intestinal metaplasia was significantly more severe $(p=0.01)$ in the antrum relative to the corpus in the cagA+ group compared with the cagA- group. No other net score differed significantly between the two groups (table 6). In keeping with this finding, when the 82 subjects were divided (on the basis of the net grade for chronic inflammation) into antral predominant (net score $>0$ ) and diffuse or pangastritis (net score $\leqslant 0$ ) categories, the prevalence of cagA+ strains was almost identical (20 of 39 and 21 of 43, respectively). In order to investigate the effect of cagA status on the pattern of gastritis separately from the influence of ulcer phenotype, 22 patients with non-ulcer dyspepsia infected with cagA- $H$ pylori (age range, 29-74 years; median, 59.5 years) were age matched with non-ulcer dyspepsia subjects infected with cagA+ strains (age range, 26-76 years; median, 59 years; $\mathrm{p}=0.9344$ ) and antral predominance sought as above. The outcome was essentially similar to that found with the subjects from mixed clinical groups (data not shown); however, for intestinal metaplasia there was a significantly greater degree of antral predominance in the cagA + group $(\mathrm{p}=0.027)$.

vacA

Unlike the cagA matched group, no antral predominance was demonstrated for any feature of the vacA s1 or s2 subgroups.

\section{Discussion}

This study is the first to investigate $H$ pylori cagA and vacA status in relation to the distribution of gastritis, a major correlate of clinical disease. While other studies have examined cagA and vacA status in relation to clinical associations and histological severity, our investigation addresses some important shortcomings of previous studies. We have investigated a sufficiently large series to yield a substantial number of cagA- subjects, thereby enabling statistically significant results to be obtained. Our patients represent a typical and well characterised population of dyspeptic subjects attending a single endoscopy unit and a wide age range is included. The majority of $H$ pylori infections commence in childhood so that, to a large extent, age reflects the duration of gastritis. Indeed, we have shown previously, in a multivariate analysis, that age is related significantly to the degree of polymorph activity, atrophy, and intestinal metaplasia, irrespective of clinical disease. ${ }^{16} \mathrm{~W}$ ith this in mind, we selected age matched subjects to make detailed comparisons of the severity and pattern of gastritis in antral and corpus biopsies according to cagA and vacA status. Finally, the pattern of gastritis is modified readily by acid suppressant drugs, ${ }^{17}$ and certain histological features, in particular surface epithelial degeneration, will be affected by concurrent NSAID use. Therefore, we eliminated subjects who had taken such drugs in the four weeks before endoscopy.

The prevalence of cagA and vacA s1 strains was significantly different in patients with duodenal ulcers compared with those with nonulcer dyspepsia $(\mathrm{p}<0.0001, \mathrm{p}=0.049$, respectively) (table 1). As in other reports, ${ }^{518}$ we also found that cagA and vacA s1 were highly significantly associated with each other $(\mathrm{p}<0.0001)$. However, when considering cagA $+H$ pylor $i$ isolates, a higher percentage was found in nonulcer dyspepsia patients $(51 \%)$ than in patients with duodenal ulcers (43\%). Therefore, our data indicates, as others have found, ${ }^{19}$ that a clinical diagnosis of duodenal ulcer is very likely to be associated with the cagA+ genotype of $H$ pylori but the presence of a cagA+/vacA s1 strain cannot be taken to predict current duodenal ulcer disease. Nevertheless, there must be a risk that some non-ulcer dyspepsia patients with cagA+ strains will develop duodenal ulceration in the future. The magnitude of this risk can only be determined by longitudinal studies. Interestingly, we found a similar prevalence of cagA+ strains in gastric ulcer and non-ulcer dyspepsia patients (58\% and $56 \%$, respectively), although the number of ulcer patients was small. Turning to the strains lacking these virulence markers, $15 \%$ of cagA- and $17 \%$ of vacA s 2 strains were 
associated with peptic ulcer disease. Thus, these genotypes alone cannot be used to select out patients with "good" or "neutral" $H$ pylori strains who need not receive eradication treatment. $^{20}$

The presence of cagA was shown to have a significant association with increased severity of chronic inflammation, polymorph activity, and surface epithelial degeneration in both the antrum and corpus. Atrophy and intestinal metaplasia were only significantly greater in the antrum (table 2). The greater degree of polymorph activity and epithelial degeneration found with cagA+ strains was not associated with increased $H$ pylori colonisation as judged by histology, despite the finding of a fourfold increase in antral colonisation density for cagA + versus cagA- strains using quantitative culture..$^{21}$ As would be expected, the prevalence of duodenal ulcers was significantly different in the cagA+ and cagA- matched subgroups. However, when matched patients were grouped according to clinical disease, regardless of strain type, other histological features emerged as significantly different. Duodenal ulcer patients exhibited a greater density of $H$ pylori colonisation in the antrum compared to age matched subjects with non-ulcer dyspepsia (who had normal findings on endoscopy), while the latter showed more severe polymorph activity and surface epithelial degeneration in the corpus than duodenal ulcer patients (table 4).

In contrast to cagA, vacA s1 was not associated with significantly higher scores for chronic inflammation, activity, atrophy, or intestinal metaplasia. However, in the light of a recently published report, one could argue that other regions of the vacA gene (subgroups of $\mathrm{s} 1$ and the middle region) may be linked more closely to histological changes and duodenal ulcer disease. ${ }^{18}$ Atherton et al found that the signal sequence s1a was associated closely with peptic ulcer disease; however, the effect of the middle region subtypes ( $\mathrm{m} 1$ and $\mathrm{m} 2$ ) on gastritis scores was not substantial. ${ }^{18}$ When we originally set out on our investigation, the s 2 genotype had been shown to be non-cytotoxic and the s1 genotype was found to produce cytotoxin, with the middle region subtypes associated with the amount of cytotoxin produced. ${ }^{5}$ Because we were concerned with the potential for a strain to produce cytotoxin, we investigated the relation between the $\mathrm{s} 1$ and s2 types only. The fact that ours is a large study should have increased the chances of finding a significant influence of the vacA signal type on the severity and pattern of gastritis. When 16 subjects with the vacA s2 genotype were age matched with s1 cases, the only statistically significant difference in histology was the greater degree of surface epithelial degeneration in the corpus in patients with the s1 genotype, a finding in keeping with cytotoxin production by this strain. Surface epithelial degeneration in the antrum and polymorph activity in the corpus were also substantially more severe in the 1 group, but the differences did not reach statistical significance (table 3 ).

It is well recognised that duodenal ulcer disease is associated with an antral predominant gastritis and this was confirmed in the present study. In order to assess and compare antral predominance we subtracted the individual grades given to the histological features in corpus mucosa from those given to the corresponding antral biopsies. Duodenal ulcer subjects had a significantly greater degree of antral predominance over non-ulcer dyspepsia patients for all the features assessed except intestinal metaplasia (table 5). However, when the same approach was applied to cagA positive and negative subjects, the only feature with significant antral predominance was intestinal metaplasia (table 6). When antral predominance was sought for vacA s1 strains, none of the features achieved significance. It was thought that the influence of virulence factors on the pattern of gastritis might be seen to better effect in patients who do not have peptic ulcers. However, when antral predominance was sought in cagA+ versus cagA- non-ulcer dyspepsia patients, a significant difference was confined to intestinal metaplasia, as with subjects from mixed clinical groups. Thus, it was concluded that in contrast to the severity of histological change, the overall pattern of gastritis is not dictated by the infecting strain, at least as indicated by cagA and vacA status. Inevitably, this conclusion points to a fundamental role for host factors in determining the outcome in terms of the gastritis phenotype and associated disease. How can we reconcile this finding with the known association between cagA positivity and both duodenal ulcer disease and gastric cancer? As we have speculated previously, ${ }^{17}{ }^{22}$ the answer probably lies in differing levels of acid production.

In patients with an enlarged parietal cell mass and high acid output, the intolerance of $H$ pylori to high local acid levels renders the corpus hostile to colonisation so that inflammation becomes concentrated in the antrum. Infection with "pro-inflammatory" cagA+/ vacA s1 strains could increase cytokine effects on antral somatostatin producing D cells and result in hypergastrinaemia. In keeping with this suggestion, serum gastrin concentrations in patients infected with cagA + strains are higher than their cagA- counterparts. ${ }^{23}$ An increased acid load reaching the first part of the duodenum leads to gastric metaplasia which will become colonised by $H$ pylori. It is reasonable to assume that by inducing more epithelial damage and inflammation, $\operatorname{cag} \mathrm{A}+/ \mathrm{vacA} \mathrm{s} 1$ strains infecting this metaplastic epithelium will result in more active chronic duodenitis ("bulbitis") than cagA- strains and, thereby, increase the risk of duodenal ulceration. This hypothesis remains to be tested, but it offers a plausible explanation for the closer association between cagA positivity and ulceration.

In subjects with normal, or subnormal levels of acid secretion, colonisation will be more evenly spread throughout the stomach. Thus, non-ulcer dyspepsia subjects show more corpus inflammation and atrophy than their age matched counterparts with duodenal ulceration. Corpus inflammation exerts cytokine effects on parietal cells, decreasing their sensitivity to gastrin so that even in the face of 
hypergastrinaemia caused by antral inflammation there is no corresponding rise in acid output. ${ }^{23}$ The appearance of multifocal atrophy principally affecting corpus mucosa and the ensuing hypochlorhydria are major risk factors for the development of gastric cancer. ${ }^{24}$ Antral atrophy does not have a direct effect on acid production and in isolation is of lesser importance as a risk factor. We have shown that cagA+ strains are associated with more atrophy and intestinal metaplasia compared with cagAstrains, and a similar association has been demonstrated between the presence of atrophy and anti-CagA antibodies. ${ }^{25}$ However, the extent of atrophy will be dependent upon the topography of the $H$ pylori gastritis. Thus, duodenal ulcer patients with antral predominant gastritis will develop antral atrophy with little or no involvement of the corpus, and be at much lower risk of gastric cancer than non-ulcer dyspepsia or asymptomatic individuals with a corpus predominant gastritis. ${ }^{26}$

In our study, cagA+ patients were almost four times more likely to have antral intestinal metaplasia than their age matched cagAcounterparts. Although we did not demonstrate significant links between vacA status and atrophy and intestinal metaplasia, an association between in vitro cytotoxic activity of $H$ pylori isolates and the presence of atrophic gastritis has been demonstrated in a population at high risk of gastric cancer in Japan. ${ }^{27}$ It is known that cytotoxic strains induce more polymorph infiltration in vivo, ${ }^{28}$ and a greater oxidative burst in polymorphs in vitro, than non-cytotoxin producers. ${ }^{29}$ Furthermore, reactive oxygen metabolites have been implicated in the pathogenesis of atrophy and DNA mutations. ${ }^{30}$ Thus, if host factors, and principally reduced acid output, permit colonisation throughout the stomach then cagA+/vacA s1 $H$ pylori strains will have a greater propensity to produce multifocal atrophy, intestinal metaplasia and, possibly, DNA damage than cagA-/ vacA s2 strains. Multifocal atrophy and intestinal metaplasia are characteristic of the gastric mucosa in which the intestinal type of gastric cancer develops. It remains to be established whether $\operatorname{cagA+/vacA}$ s1 strains increase free radical damage to DNA in gastric epithelial cells or increase epithelial cell proliferation relative to cagA-/vacA s2 strains and, thereby, exert a more direct role in gastric carcinogenesis.

In conclusion, this study has shown that while the possession of the cagA and vacA s1 genotype is linked to the severity of chronic $H$ pylori gastritis these putative virulence markers do not appear to determine its overall pattern. In so far as the pattern of gastritis is strongly linked to the nature of the associated disease, our findings indicate that host or environmental factors largely determine the type of disease that will complicate long standing $H$ pylori infection, while the magnitude of the risk of developing the disease is related to bacterial virulence factors.

1 Figura N, Guglielmetti P, Rossolini, et al. Cytotoxin production by Campylobacter pylori strains isolated fom patients tion by Campylobacter pylori strains isolated fom patients
with peptic ulcers and from patients with chronic gastritis with peptic ulcers and from patients
only. $\mathcal{F}$ Clin Microbiol 1989;27:225-6.
2 Cover TL, Cao P, Lind CD, et al. Correlation between vacuolating cytotoxin production by Helicobacter pylori isolates in vitro and in vivo. Infect Immun 1993;61:5008-12.

3 Phadnis SH, Ilver D, Janzon L, et al. Pathological significance and molecular characterisation of the vacuolating toxin gene of Helicobacter pylori. Infect Immun 1994;62:1557-65.

4 Cover TL, Tummuru MKR, Cao P, et al. Divergence of genetic sequences for the vacuolating cytotoxin among Helicobacter pylori strains. $f$ Biol Chem 1994;269:1056673 .

5 Atherton JC, Cao P, Peek RM, et al. Mosaicism in vacuolating cytotoxin alleles of Helicobacter pylori. f Biol Chem 1995;270:17771-7.

6 Cover TL, Dooley CP, Blaser MJ. Characterisation of and human serologic response to proteins in Helicobacter pylori broth culture supernatants with vacuolizing cytotoxin activity. Infect Immun 1990;58:603-10.

7 Tummuru MKR, Cover TL, Blaser MJ. Cloning and expression of a high-molecular-mass major antigen of Helicobacter pylori: evidence of linkage to cytotoxin production. Infect Immun 1993;61;1799-809.

8 Censini S, Lange C, Xiang Z, et al. Cag A pathogenicity island (PAI) of Helicobacter pylori encodes for type I-specific virulence factors [abstract]. Gut 1996;39(suppl 2):A72.

9 Crabtree JE, Farmery SM, Lindley IJD, et al. CagA/ cytotoxic strains of Helicobacter pylori and interleukin-8 in gastric epithelial cell lines. F Clin Pathol 1994;47:945-50.

10 Cover TL, Glupczynski Y, Lage AP, et al. Serologic detection of infection with $\mathrm{cagA}^{+}$Helicobacter pylori strains. F Clin Microbiol 1995;33:1496-500.

11 Ching CK, Wong BC, Kwok E, et al. Prevalence of CagA-bearing Helicobacter pylori strains detected by the anti-CagA assay in patients with peptic ulcer disease and in controls. Am f Gastroenterol 1996;91:949-53.

12 Crabtree JE, Wyatt JI, Sobala G, et al. Systemic and mucosal humoral responses to Helicobacter pylori in gastric cancer. Gut 1993;34:1339-43.

13 Parsonnet J, Friedman GD, Orentreich N, et al. Risk for gastric cancer in people with CagA positive or CagA negative Helicobacter pylori infection. Gut 1997;40:297-301.

14 Dixon MF, Genta RM, Yardley JH, et al. Classification and grading of gastritis: the updated Sydney System. Am F Surg Pathol 1996;20:1161-81.

15 Ho S-A, Hoyle JA, Lewis FA, et al. Direct polymerase chain reaction test for detection of Helicobacter pylori in humans and animals. F Clin Microbiol 1991;29:2543-9.

16 Sobala GM, Axon ATR, Dixon MF. Morphology of chronic antral gastritis: relationship to age, Helicobacter pylori status and peptic ulceration. Eur f Gastroenterol Hepatol 1992; 4:825-9.

17 Lee A, Dixon MF, Danon SJ, et al. Local acid production and Helicobacter pylori: a unifying hypothesis of gastroduodenal disease. Eur $\mathcal{F}$ Gastroenterol Hepatol 1995;7: $461-5$.

18 Atherton JC, Peek RM, Tham KT, et al. Clinical and pathological importance of heterogeneity in vacA, the vacuolating cytotoxin gene of Helicobacter pylori. Gastroenterology 1997;112:92-9.

19 Go MF, Graham DY. Presence of the cagA gene in the majority of Helicobacter pylori strains is independent of whether the individual has duodenal ulcer or asymptomatic gastritis. Helicobacter 1996;1:107-11.

20 Blaser MJ. Not all Helicobacter pylori strains are created equal: should all be eliminated? Lancet 1997;349:1020-2.

21 Atherton JC, Tham KT, Peek RM, et al. Density of Helicobacter pylori infection in vivo as assessed by quantitative bacter pylori infection in vivo as assessed by quantit
culture and histology. F Infect Dis 1996;174:552-6.

22 Dixon MF. Helicobacter pylori and peptic-ulceration - histopathological aspects. F Gastroenterol Hepatol 1991;6:12530

23 McColl KEL, El-Omar E, Gillen D, et al. H. pylori-induced hypergastrinaemia is related to bacterial CagA status [abstract]. Gut 1997;40(suppl 1):A46.

24 Correa P. Human gastric carcinogenesis: a multistep and multifactorial process-first American Cancer Society award lecture on cancer epidemiology and prevention. award lecture on cancer ep

25 Beales IL, Crabtree JE, Scunes D, et al. Antibodies to CagA protein are associated with gastric atrophy in Helicobacter pylori infection. Eur f Gastroenterol Hepatol 1996;8:645-9.

26 Miehlke S, Bayerdörffer E, Meining A, et al. Identifying persons at risk of gastric cancer. Helicobacter 1997;2(supp1 1):61-6.

27 Ito S, Azuma T, Murakita $\mathrm{H}$, et al. Profile of Helicobacter pylori cytotoxin derived from two areas of Japan with different prevalence of atrophic gastritis. Gut 1996;39:800-6.

28 Rautelin H, Sipponen P, Sepppala K, et al. Gastric inflammation and neutrophil-activating and cytotoxinproducing Helicobacter pylori strains. Scand $\mathcal{F}$ Gastroenterol 1996;31:639-42.

29 Zhang QB, Nakashabendi M, Mokhashi MS, et al. Association of cytotoxin production and neutrophil activation by strains of Helicobacter pylori isolated from patients with peptic ulceration and chronic gastritis. Gut 1996;38: $841-5$.

30 Goldstone AR, Quirke P, Dixon MF. Helicobacter pylori and gastric cancer. F Pathol 1996;179:129-37. 